Supplementary Information for

Using Magnetometry to Understand the Relative Role of Magnetic Particles in Co-based Catalysts for the Oxygen Reduction Reaction

Anne Marie Esposito ${ }^{1}$, Alexis Magaña ${ }^{1}$, Eric S. Thornburg ${ }^{1}$, Richard T. Haasch ${ }^{2}$, Hong Yang ${ }^{3}$, and Andrew A. Gewirth ${ }^{1,4 *}$

${ }^{1}$ Department of Chemistry, University of Illinois, Urbana, IL 61801, USA

${ }^{2}$ Frederick Seitz Materials Research Laboratory, University of Illinois, Urbana, Illinois 61801, USA

${ }^{3}$ Department of Chemical and Biomolecular Engineering, University of Illinois, Urbana, IL 61801, USA

${ }^{4}$ International Institute for Carbon Neutral Energy Research (WPI-I2CNER), Kyushu University, Fukuoka, Fukuoka 819-0385, Japan

*author to whom correspondence should be addressed. Email: agewirth@illinois.edu, Tel: 217333-8329 
Table S1. Summary of half-wave potentials and number of electrons transferred for all materials as measured in $0.1 \mathrm{M} \mathrm{HClO}_{4}$.

\begin{tabular}{|l|l|l|l|}
\hline Sample & $\mathrm{E}_{1 / 2}(\mathrm{~V})$ & $\mathrm{n}$ & $\% \mathrm{H}_{2} \mathrm{O}_{2}$ \\
\hline CoNC as-prepared & $0.71 \pm 0.01$ & $3.8 \pm 0.1$ & $8 \pm 6$ \\
\hline CoNC acid washed & $0.69 \pm 0.02$ & $3.78 \pm 0.06$ & $5 \pm 2$ \\
\hline CoNC-CTAB as-prepared & $0.59 \pm 0.04$ & $3.8 \pm 0.1$ & $12 \pm 5$ \\
\hline CoNC-CTAB acid washed & $0.68 \pm 0.03$ & $3.70 \pm 0.07$ & $15 \pm 4$ \\
\hline
\end{tabular}

Table S2. Summary of half-wave potentials and number of electrons transferred for all materials as measured in $0.1 \mathrm{M} \mathrm{NaOH}$.

\begin{tabular}{|l|l|l|l|}
\hline Sample & $\mathrm{E}_{1 / 2}(\mathrm{~V})$ & $\mathrm{n}$ & $\% \mathrm{OH}_{2}^{-}$ \\
\hline CoNC as-prepared & $0.74 \pm 0.01$ & $3.76 \pm 0.08$ & $12 \pm 4$ \\
\hline CoNC acid washed & $0.77 \pm 0.02$ & $3.2 \pm 0.1$ & $39 \pm 7$ \\
\hline CoNC-CTAB as-prepared & $0.71 \pm 0.04$ & $3.7 \pm 0.2$ & $16 \pm 8$ \\
\hline CoNC-CTAB acid washed & $0.77 \pm 0.01$ & $3.7 \pm 0.1$ & $13 \pm 5$ \\
\hline Co NPs + XC72 & $0.73 \pm 0.03$ & $3.3 \pm 0.2$ & $36 \pm 12$ \\
\hline
\end{tabular}

Table S3. Summary of the reaction rate constant $\left(\mathrm{k}_{\mathrm{f}}\right)$ of materials as measured in $0.1 \mathrm{M} \mathrm{NaOH}$ at $0.720 \mathrm{~V}$ vs. RHE.

\begin{tabular}{|l|l|l|l|l|}
\hline Material & $\begin{array}{l}\text { Rate Constant } \\
\left(10^{-6} \mathrm{~cm} \mathrm{~s}^{-1}\right)\end{array}$ & $\begin{array}{l}\text { Rate } \\
\text { Constant/atom } \\
\left(10^{-23}\right)\end{array}$ & $\begin{array}{l}\text { Rate } \\
\text { Constant/Particle } \\
\left(10^{-23}\right)\end{array}$ & $\begin{array}{l}\text { Tafel Slope } \\
(\mathrm{mV} / \mathrm{dec})\end{array}$ \\
\hline CoNC & $1.1 \pm 0.5$ & $1.9 \pm 0.9$ & NA & $60 \pm 9$ \\
\hline $\begin{array}{l}\text { Co NPs }+ \\
\text { XC72 }\end{array}$ & $0.9 \pm 0.5$ & $0.5 \pm 0.2^{\mathrm{a}}$ & $228 \pm 116$ & $54 \pm 6$ \\
\hline
\end{tabular}

${ }^{\mathrm{a} C a l c u l a t e d ~ f o r ~ s u r f a c e}$ atoms of the particle only

\section{Calculation S1}

The rate constant was calculated using a Koutecky-Levich analysis. $1 / \mathrm{i}$ vs. $\omega^{-0.5}$ was plotted with the current at the given potential to yield a y-intercept of $1 / i_{K} \cdot i_{K}$ was then used to calculate the $\mathrm{k}_{\mathrm{f}}$ for each catalyst. The following equations were used ${ }^{1-2}$ :

$$
\frac{1}{\bar{l}}=\frac{1}{i_{k}}+\frac{1}{0.62 n F A D^{2 / 3} \omega^{\frac{1}{2}} v^{-\frac{1}{6}} C_{O_{2}}}
$$


Where $\mathrm{n}$ is the number of electrons transferred as determined by RRDE measurements, $\mathrm{F}$ is Faraday's constant, A is the geometric electrode area, D is the diffusion coefficient, $\omega$ is the rotation rate, $v$ is the scan rate, $\mathrm{C}_{\mathrm{O} 2}$ is the concentration of $\mathrm{O}_{2}, \mathrm{i}$ is the current and $\mathrm{i}_{\mathrm{K}}$ is the kinetic current. $\quad i_{k}=F A k_{f} C_{O_{2}} e^{\frac{-\alpha F\left(E-E_{0}\right)}{R T}}$

Where $\mathrm{k}_{\mathrm{f}}$ is the rate constant, $\alpha$ is the transfer coefficient (assumed to be 0.5 ), $\mathrm{E}$ is the potential that the kinetic analysis is performed at, $\mathrm{E}_{0}$ is the equilibrium potential, $\mathrm{R}$ is the gas constant and $\mathrm{T}$ is temperature.

\section{Calculation S2}

We determined the number of Co atoms present in a single electrode cast for the catalysts using the ICP-OES data (Table 1) and the mass of catalyst on the electrode. We assume that all Co atoms present in the cast are active.

From the SQUID magnetometry measurements of the Co NPs + XC72 catalyst (Fig. S5) we can determine the volume of the Co nanoparticles by a Langevin function $(\mathcal{L})$ fit given by Equation $2: 3-5$

$$
\frac{M}{M_{\text {sat }}}=\mathcal{L}\left(\frac{M_{s} V H}{k_{B} T}\right)
$$

where $M$ is equal to the magnetization, $M_{\text {sat }}$ is equal to the saturation magnetization, $M_{s}$ is equal to the spontaneous magnetization determined for $\mathrm{Co}^{0}\left(1335 \mathrm{emu} / \mathrm{cm}^{3}\right), V$ is equal to the volume of a particle, $H$ is equal to the applied magnetic field, $\mathrm{k}_{\mathrm{B}}$ is the Boltzmann constant, and $T$ is the temperature. The average number of atoms in a single Co nanoparticle was calculated by determining the value of $V$ for each sample and assuming spherical Co particles. The volume of each particle allowed us to calculate the number of Co atoms present per particle. Based on ICP- 
OES data and percentage of non-paramagnetic Co (Table 1) and the mass of catalyst cast on the electrode we can determine the total number of particles. Based on the diameter of a Co atom, we can calculate the number of atoms that would be on the surface of the particle.

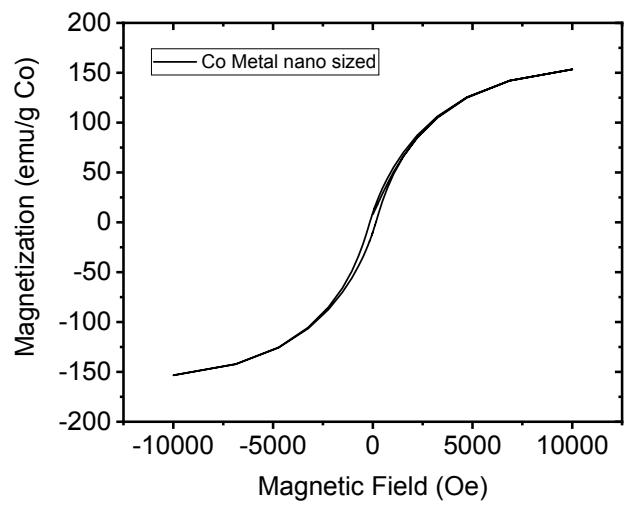

Figure S1. The effect of an applied magnetic field on magnetization of nanosized Co metal particles.

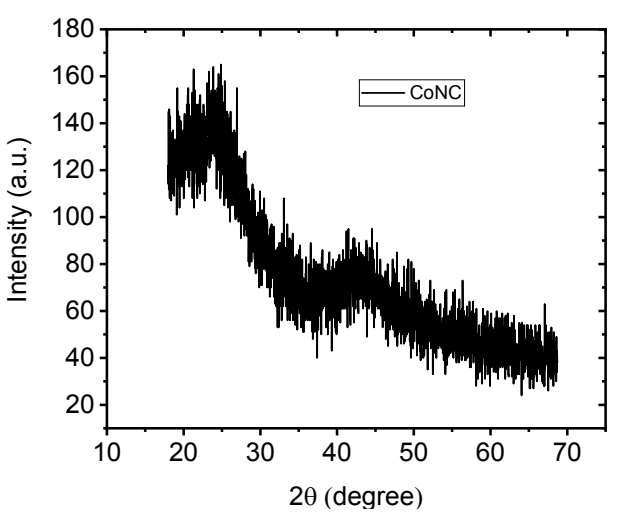

Figure S3. The XRD pattern for CoNCCTAB as-prepared.

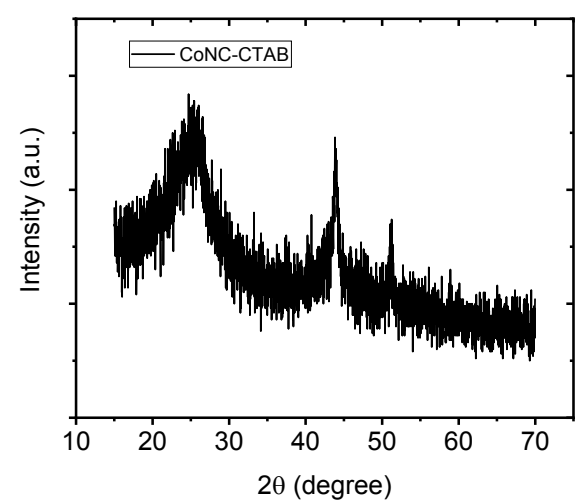

Figure S2. The XRD pattern for CoNCCTAB as-prepared.

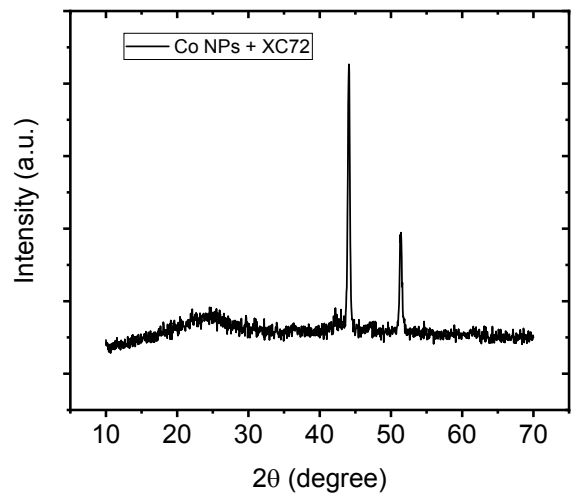

Figure S4. The XRD pattern for Co NPs + XC72 catalyst. 


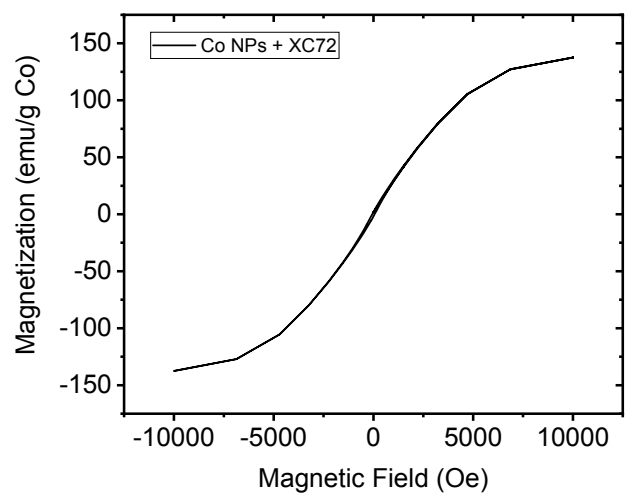

Figure S5. The effect of an applied magnetic field on magnetization of Co NPs + XC72.

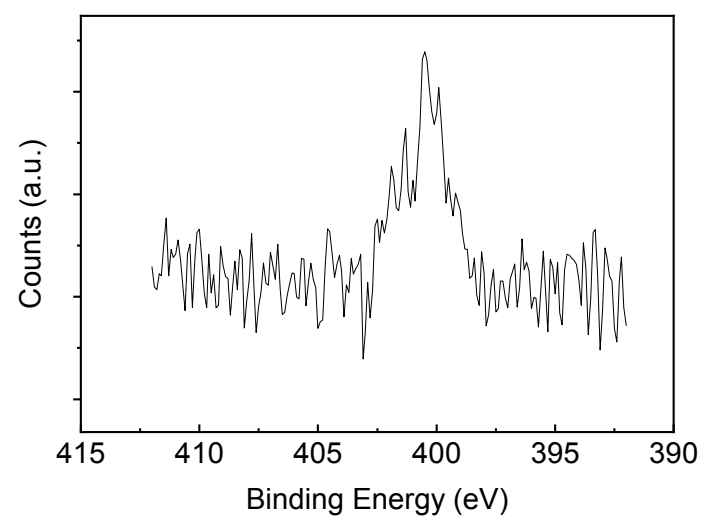

Figure S7. The N 1s XPS of Co NPs + XC72.

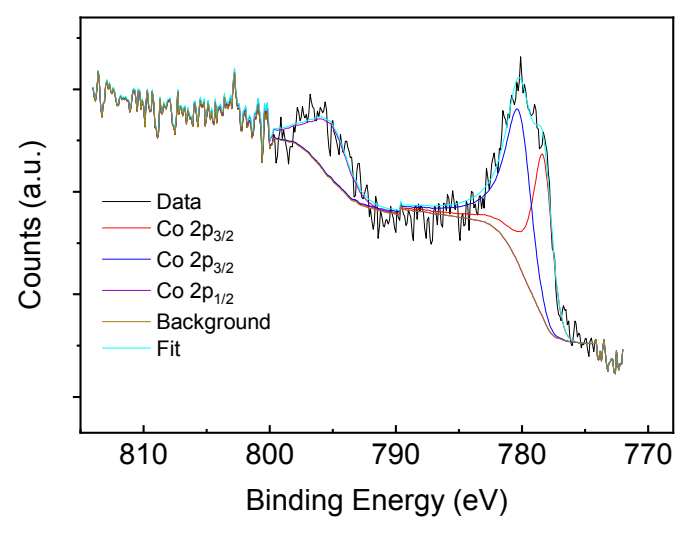

Figure S6. The Co $2 p$ XPS of Co NPs + XC72. 

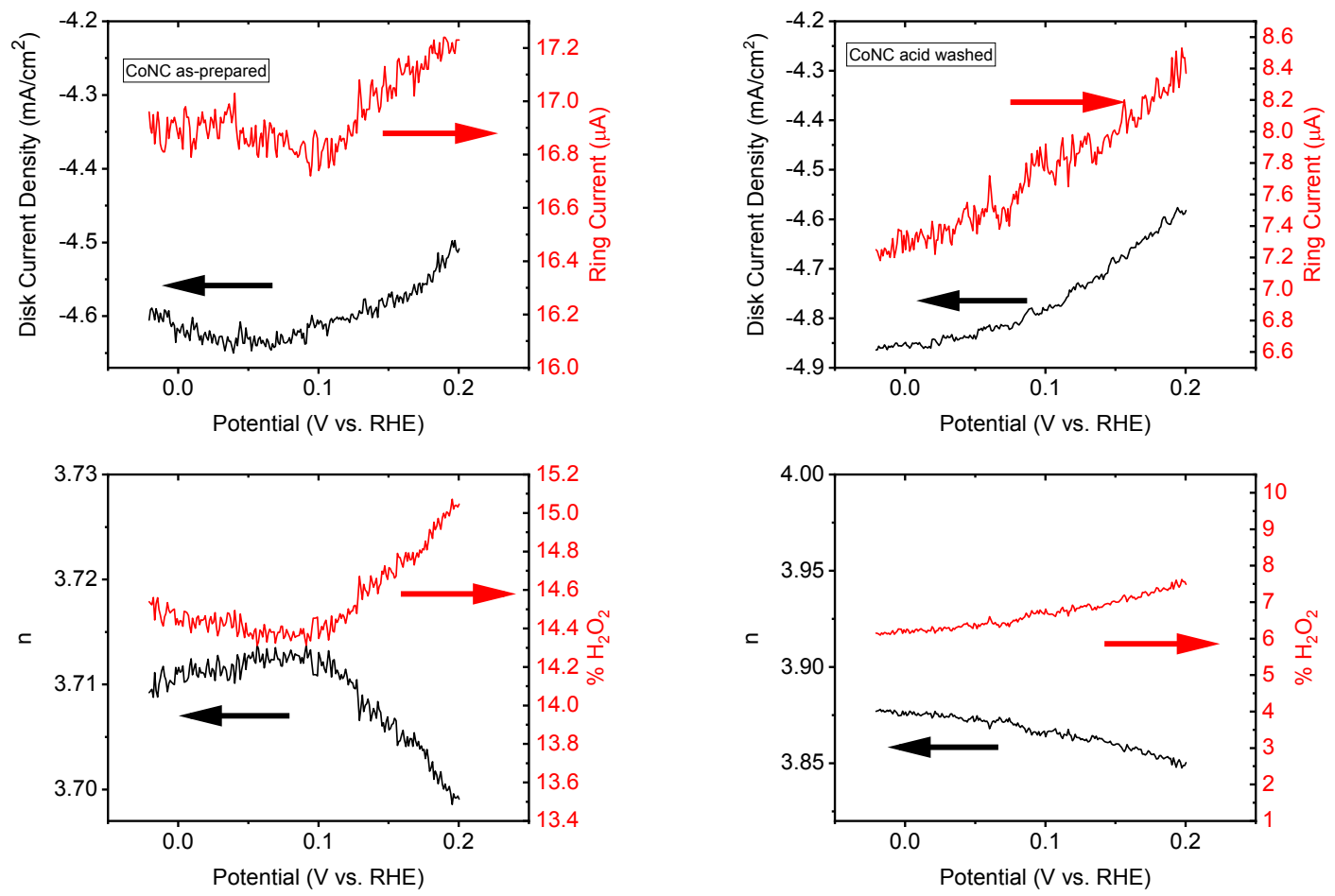

Figure S8. Representative disk and ring current collected for the CoNC as-prepared and CoNC acid washed materials measured in $0.1 \mathrm{M} \mathrm{HClO}_{4}$. Bottom graphs are the calculated $\mathrm{n}$ and $\% \mathrm{H}_{2} \mathrm{O}_{2}$ values for the potential regions shown.
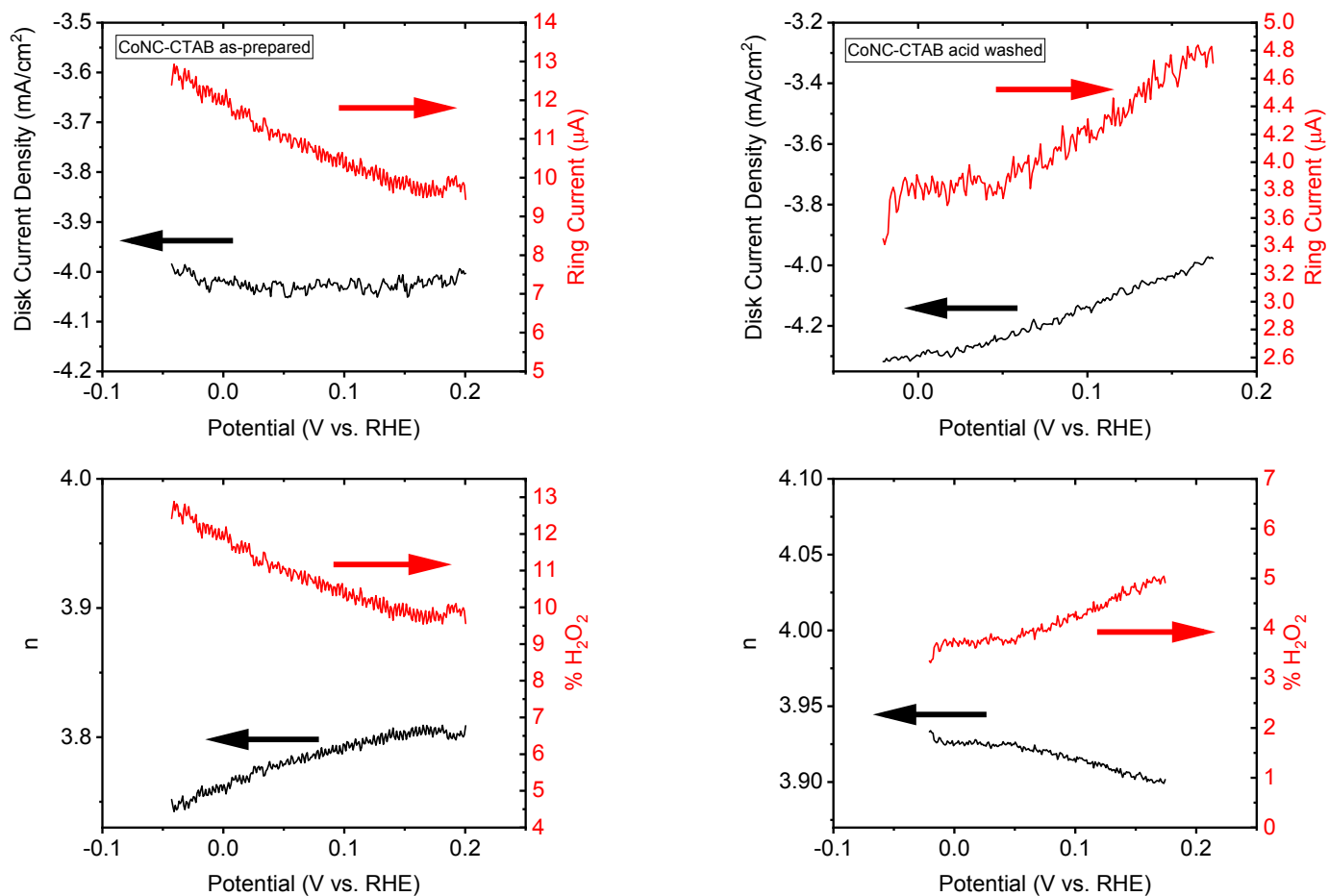

Figure S9. Representative disk and ring current collected for the CoNC-CTAB as-prepared and CoNC-CTAB acid washed materials measured in $0.1 \mathrm{M} \mathrm{HClO}_{4}$. Bottom graphs are the calculated $\mathrm{n}$ and $\% \mathrm{H}_{2} \mathrm{O}_{2}$ for the potential regions shown. 

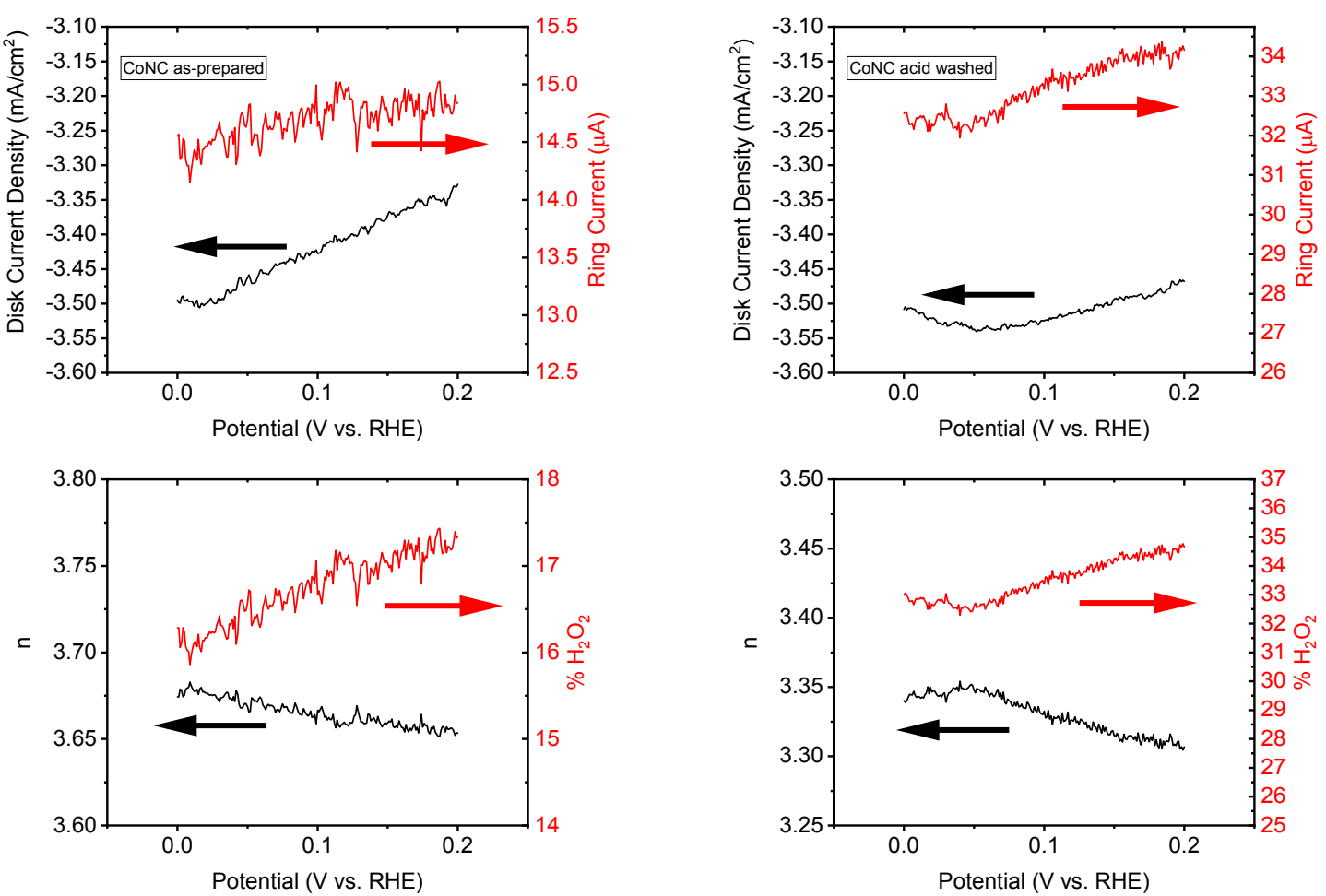

Figure S10. Representative disk and ring current collected for the CoNC as-prepared and CoNC acid washed materials measured in $0.1 \mathrm{M} \mathrm{NaOH}$. Bottom graphs are the calculated $\mathrm{n}$ and $\% \mathrm{H}_{2} \mathrm{O}_{2}$ values for the potential regions shown.
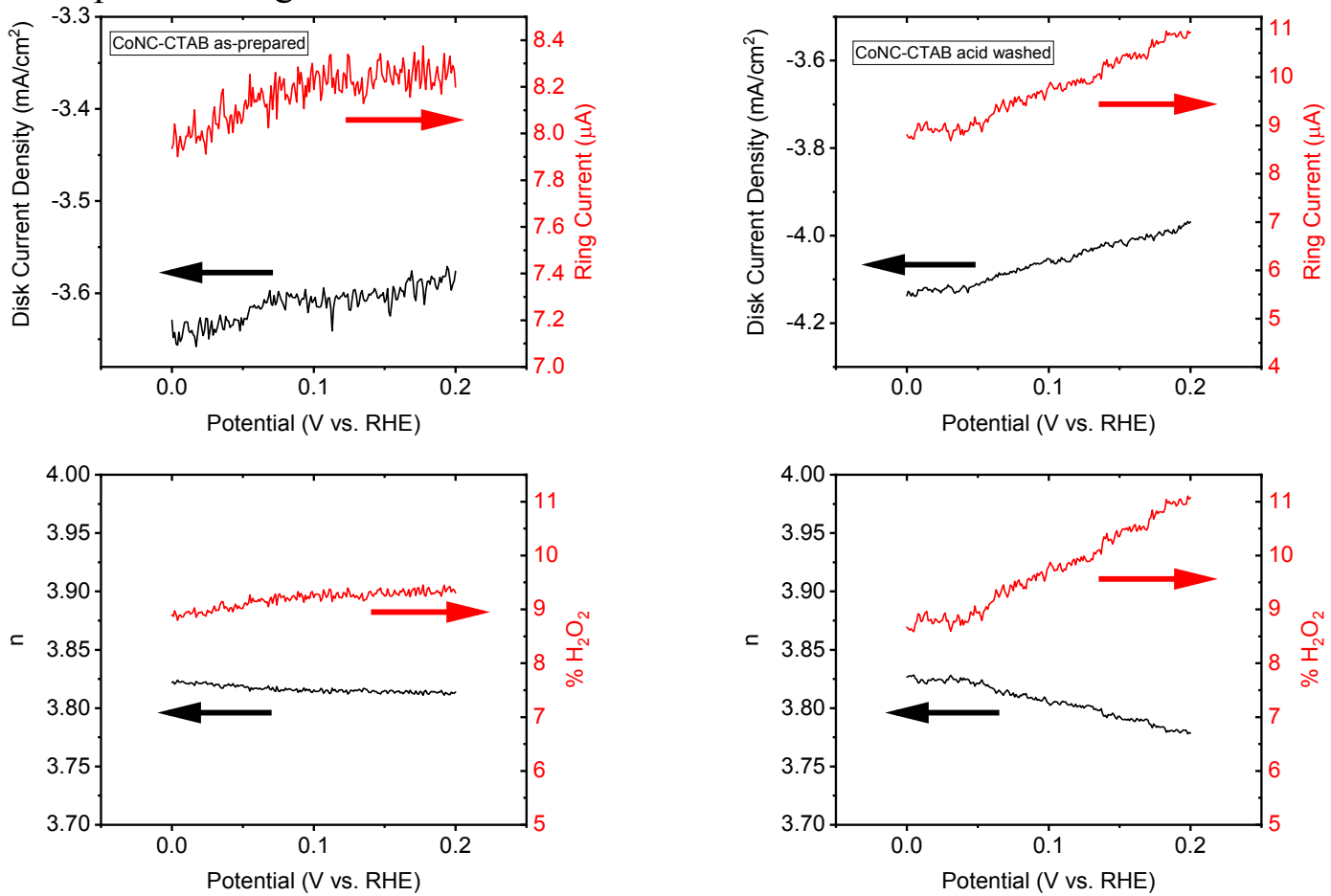

Figure S11. Representative disk and ring current collected for the CoNC-CTAB as-prepared and CoNC-CTAB acid washed materials measured in $0.1 \mathrm{M} \mathrm{NaOH}$. Bottom graphs are the calculated $\mathrm{n}$ and $\% \mathrm{H}_{2} \mathrm{O}_{2}$ values for the potential regions shown. 


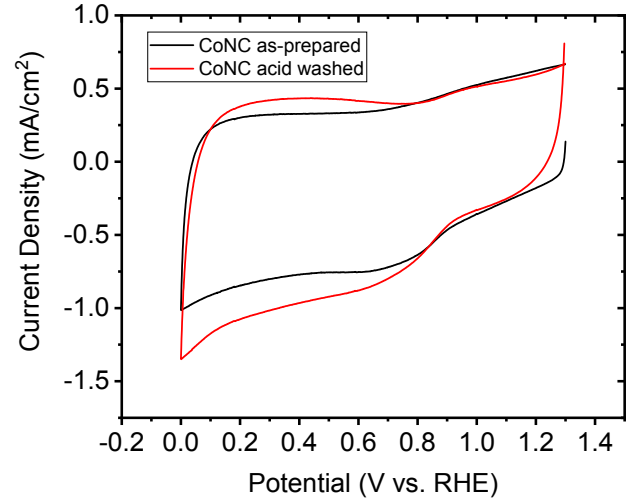

Figure S12. Representative CVs of CoNC materials in Ar-saturated $0.1 \mathrm{M} \mathrm{NaOH}$ electrolyte.

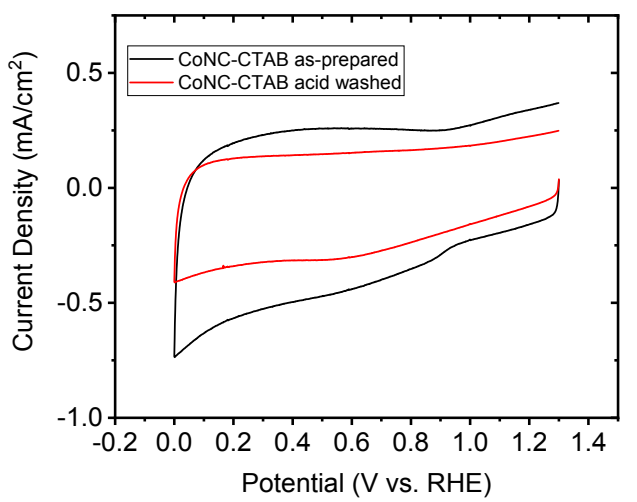

Figure S13. Representative CVs of CoNC materials in Ar-saturated 0.1 M NaOH electrolyte.

1. Bard, A. J.; Faulkner, L. R., Electrochemical Methods: Fundamentals and Applications. 2 ed.; John Wiley \& Sons: 2001; p 833.

2. Hsueh, K.-L.; Gonzalez, E. R.; Srinivasan, S., Electrolyte Effects on Oxygen Reduction Kinetics at Platinum: A Rotating Ring-Disk Electrode Analysis. Electrochimica Acta 1983, 28 (5), 691-97.

3. $\quad$ Brunsman, E. M.; Sutton, R.; Bortz, E.; Kirkpatrick, S.; Midelfort, K.; Williams, J.; Smith, P.; McHenry, M. E.; Majetich, S. A.; Artman, J. O.; De Graef, M.; Staley, S. W., Magnetic properties of carbon-coated, ferromagnetic nanoparticles produced by a carbon-arc method. Journal of Applied Physics 1994, 75 (10), 5882-5884.

4. $\quad$ Yang, C.; Xing, J.; Guan, Y.; Liu, J.; Liu, H., Synthesis and characterization of superparamagnetic iron nanocomposites by hydrazine reduction. Journal of Alloys and Compounds 2004, 385 (1-2), 283-287.

5. $\quad$ Mamtani, K.; Singh, D.; Tian, J.; Millet, J. M. M.; Miller, J. T.; Co, A. C.; Ozkan, U. S., Evolution of N-Coordinated Iron-Carbon (FeNC) Catalysts and Their Oxygen Reduction (ORR) Performance in Acidic Media at Various Stages of Catalyst Synthesis: An Attempt at Benchmarking. Catalysis Letters 2016, 146 (9), 1749-1770. 